\title{
Vulvar microinvasive squamous cell carcinoma arising in vulvar intraepithelial neoplasia 3 complicated by genital warts and systemic lupus erythematosus: a case report
}

\author{
Saori Seo ${ }^{1}$, Ken-ichi Honda ${ }^{1}$, Yusuke Nakano ${ }^{1}$, Naohiko Umesaki ${ }^{1}$, Yoko Iwasa ${ }^{2}$, Isao Tanaka ${ }^{2}$, \\ Yoshihito Iwai ${ }^{3}$, Tomoyo Yasui $^{1}$, Toshiyuki Sumi ${ }^{1}$, Masayasu Koyama ${ }^{1}$
}

\author{
${ }^{1}$ Department of Obstetrics and Gynecology, Osaka City University Graduate School of Medicine. Department of \\ Gynecology, Izumi Municipal Hospital, Osaka, Japan \\ ${ }^{2}$ Department of Pathology, Izumi Municipal Hospital, Osaka, Japan \\ ${ }^{3}$ Department of Urology, Izumi Municipal Hospital, Osaka, Japan
}

Received: 17 May 2013

Accepted: 15 June 2013

*Correspondence:

Dr. Saori Seo,

E-mail: m2049125@med.osaka-cu.ac.jp

(C) 2013 Seo S et al. This is an open-access article distributed under the terms of the Creative Commons Attribution Non-Commercial License, which permits unrestricted non-commercial use, distribution, and reproduction in any medium, provided the original work is properly cited.

\begin{abstract}
A patient suffering from long-term systemic lupus erythematosus attended with a complaint of recurrent genital warts. Perineal white-colored skin and a peri-anal papillary protrusion adjacent to the genital warts were biopsied and determined to be vulvar intraepithelial neoplasia (VIN) 3 and microinvasive squamous cell carcinoma (SCC), respectively. These lesions were locally excised. Human papillomavirus (HPV)-6 was detected in these lesions, including in the genital warts, while HPV-56 was detected only in the perineal VIN3 and peri-anal microinvasive SCC.
\end{abstract}

Keywords: Vulvar intraepithelial neoplasia, Genital warts, Human papillomavirus microinvasive squamous cell carcinoma, Systemic lupus erythematosus

\section{INTRODUCTION}

Vulvar intraepithelial neoplasia (VIN) and vulvar cancer are increasing in prevalence. Risk factors for these conditions include systemic factors and other vulvar diseases. ${ }^{1}$ Persistent infection with human papillomavirus (HPV), especially HPV-16, is also a major risk factor for the development of vulvar neoplasia. ${ }^{2}$ We report a case of microinvasive squamous cell carcinoma (SCC) arising in VIN3, complicated by long-term systemic lupus erythematosus and genital warts.

\section{CASE REPORT}

A 71-year-old multipara woman attended our clinic with a complaint of recurrent genital warts. She had been receiving prednisolone for systemic lupus erythematosus for 34 years. She had also suffered from recurrent genital warts for 20 years, which failed to respond to application of Imiquimod cream.

Visual examination identified genital warts measuring about $7 \mathrm{~mm}$ on the right perineum (arrow, Figure 1), which extended to the external urethral orifice. The introitus surrounding the clitoris was white in color with a rough surface (arrowheads, Figure 1) and pathological examination of the biopsy specimen revealed carcinoma in situ. A 12-mm papillary protrusion was found on the right side of the anus (circle, Figure 1) and pathological examination of the biopsy demonstrated SCC.

Excision of the warts on the right perineum and urethral orifice revealed genital warts, and excision of introitus tissue, together with several millimeters of surrounding skin, showed genital warts with VIN3 (Figure 2). The papillary tumor was removed with a $1-\mathrm{cm}$ margin of 
surrounding skin and was found to be composed of a surface layer of carcinoma in situ (Figure 3A) and focally microinvasive SCC (Figure 3B). DNA was extracted from the genital warts on the right perineum, the VIN3 on the introitus, and the microinvasive SCC using a Gentra Puregene tissue kit (Qiagen, Gaithersburg, MD, USA).

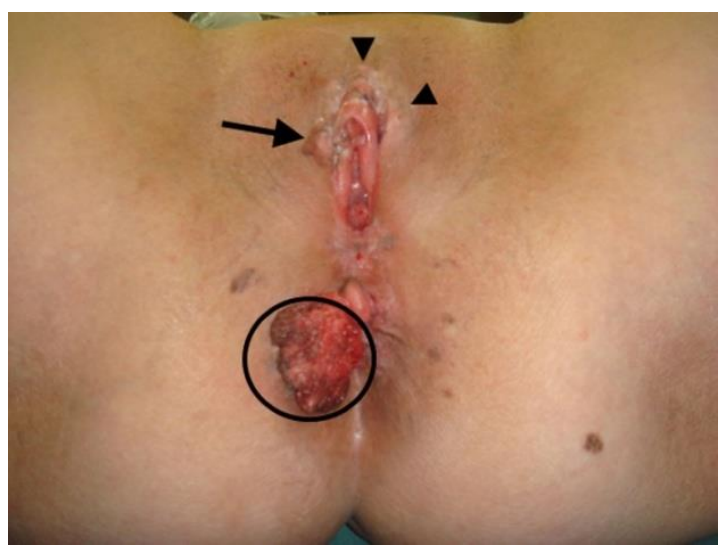

Figure 1: Genital warts (arrow), carcinoma in situ (arrowheads) on introitus and microinvasive SCC on right side skin of anus (circle).
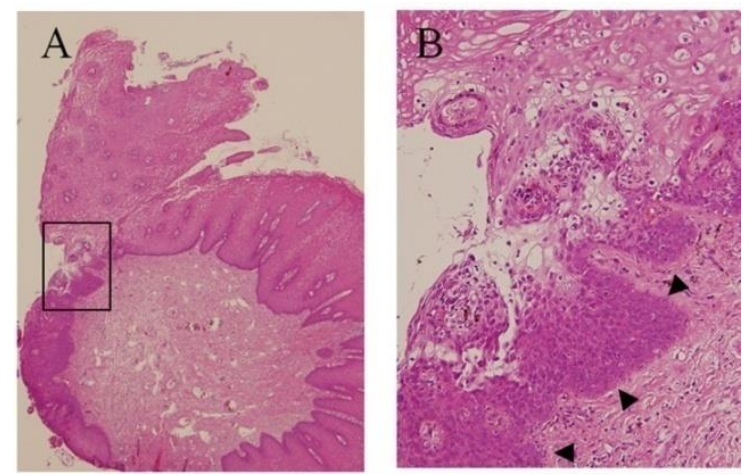

Figure 2: Microscopic findings of VIN3 in genital warts (A) and expanding front of VIN3 (B).
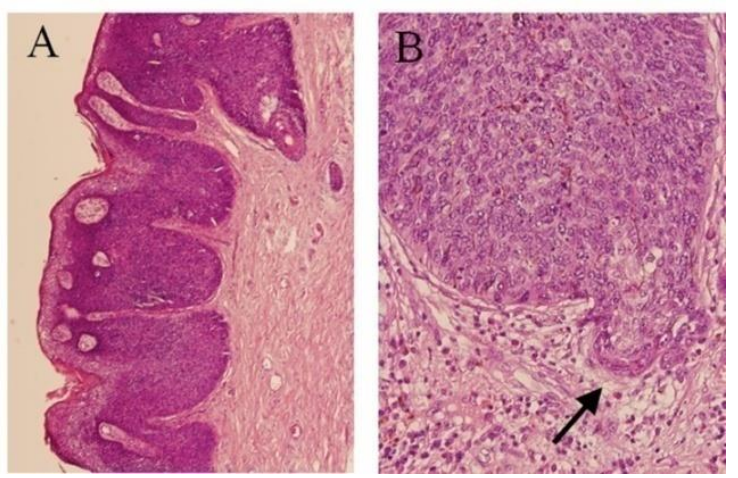

Figure 3: Microscopic findings of microinvasive SCC on peri-anal skin. Epithelial layer replaced by broad intraepithelial neoplasia (A) with focal microinvasion (B).
Four months later, genital warts reappeared on the left peri-anal skin and were excised, but no carcinoma or VIN was found. Cytology of the uterine cervix indicated a low-grade squamous intraepithelial lesion, but no dysplastic lesion was detected by colposcopy. Six months later, the genital warts recurred on the left peri-anal skin, but no further growth was detected

Linear-array analysis revealed HPV-6 in the genital warts on the right perineum, and both HPV-6 and HPV-56 in the VIN3 on the introitus and microinvasive SCC on the right peri-anal skin. The presence of HPV-6 and HPV-56 genes was confirmed by polymerase chain reaction (PCR) and DNA sequencing of the long control region of HPV-6 was examined. Two regions of the long control regions (bases 7058-7668 and 7584-8029) of HPV-6 were amplified using the following primers: forward: ATCCTTTGGGACGCAAGTTTT, and reverse: GATGCACTTGGCAAGCAACTT; and forward: TTCCACCAATTTGTTACAACG, and reverse: TGCCTCGTTTGCTAAATTTTAG, respectively. The DNA sequences of these regions were identical to those of the long control region of HPV-6 reported as HPV6W50 isolated from benign lesions. ${ }^{3}$

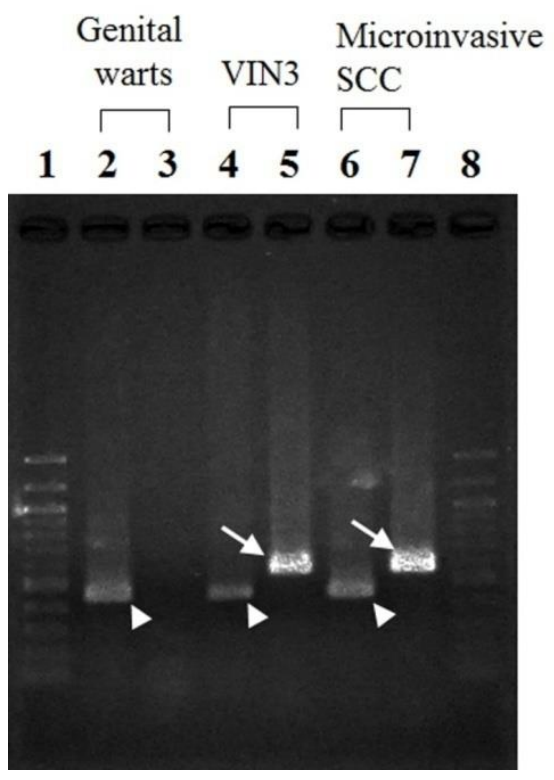

Figure 4: PCR of HPV-6 DNA (lanes 2, 4, 6) and HPV-56 DNA (lanes 3, 5, 7) from perineal genital warts (lanes 2,3), introitus VIN3 (lanes 4, 5) and perianal microinvasive SCC (lanes 6, 7) separated by agarose gel electrophoresis. Ladder marker with $\mathbf{1 0 0}$, $200,300,400,500,600,700,800,900,1000,1200,1500$ bp shown at both sides (lanes 1, 8). PCR products of HPV-6 DNA (arrowheads) were detected in all tissues, and those of HPV-56 DNA (arrows) were detected in VIN3 and microinvasive SCC. 
A

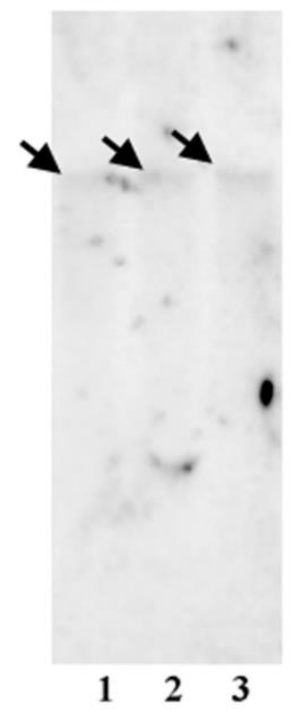

B

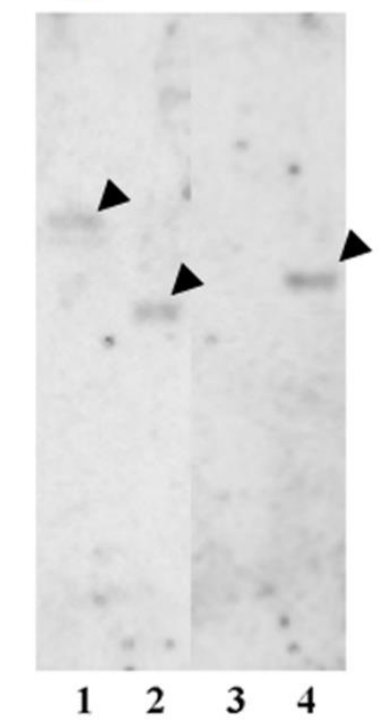

Figure 5: (A) Southern blot analysis with HPV-6 long control region probe for DNA digested by BamH1 (which has one cleavage site in HPV-6) revealed the same size DNA (indicated by arrows) in genital warts (lane 1), introitus VIN3 (lane 2) and peri-anal microinvasive SCC (lane 3). (B) Southern blot analysis with HPV-56 E1 region probe for DNA digested by

Hind III (no cleavage site in HPV-56) in VIN3 (lane 1) and peri-anal microinvasive SCC (lane 2) revealed different size DNAs (arrowheads). DNA digested by Sph I (one cleavage site in HPV-56) revealed no band in VIN3 (lane 3) and one band in peri-anal microinvasive SCC (lane 4).

For HPV-56, the E1 region of 564 bp was amplified using the following primers: forward: ACATATCCATGGAGCCACAA, and reverse: AATTGCTCATTGCACTGTAGG. The DNA sequence of this region was identical to that of HPV $-56 .{ }^{8} \mathrm{HPV}-6$, but not HPV-56, was detected in the PCR products from genital warts (Figure 4). However, both HPV-6 and HPV56 genes were detected in PCR products from VIN3 and microinvasive SCC (Figure 4).

Southern blot analysis was used to examine the integration of HPV DNA into cellular DNA using the HPV-6 long control region probe and HPV-56 E1 region probe with a DIG High Prime DNA Labeling and Detection Kit (Roche Diagnostics, Mannheim, Germany). Bam HI (with one cleavage site in HPV-6) digests showed the same mobility of HPV-6 gene in gel electrophoresis, indicating the presence of an episome. Using the HPV-56 probe, the different mobilities of the DNA were shown between microinvasive SCC and VIN3 in Hind III digests (no cleavage site in HPV-56), and Sph I digests (one cleavage site in HPV-56), indicating integration of the HPV-56 gene into the cellular DNA.

\section{DISCUSSION}

HPVs are reported to be responsible for $38-75 \%$ of vulvar SCCs, ${ }^{4,5}$ and $84 \%$ of VIN. ${ }^{6} \mathrm{HPV}-16$ is the commonest type of HPV identified in vulvar SCCs and precancerous lesions. ${ }^{7}$ HPV- 6 and HPV-56 genes were detected in the current case, and integration of HPV-56 into the cellular DNA was detected in VIN and microinvasive SCC, but not in the genital warts. It was therefore considered that HPV-56 infection was responsible for inducing carcinogenesis, ${ }^{8}$ but the depth of tumor invasion remained in the microinvasion category.

Previous reports have suggested that virus-induced malignant diseases occur more frequently in patients with systemic lupus erythematosus compared with the normal population. ${ }^{8}$ Therapeutic drugs, including prednisolone, are also thought to reduce the innate immune response to HPV infection by down-regulating toll-like receptors. ${ }^{9}$ Long-term therapy with prednisolone may therefore have suppressed the nucleic acid-sensing mechanisms that normally protect against virus infection in the current patient.

\section{Funding: No funding sources Conflict of interest: None declared \\ Ethical approval: Not required}

\section{REFERENCES}

1. Judson PL, Habermann EB, Baxter NN, Durham SB, Virnig BA. Trends in the incidence of invasive and in situ vulvar carcinoma. Obstet Gynecol 2006;107:1018-1022.

2. Wallbillich JJ, Rhodes HE, Milbourne AM, Munsell MF, Frumovitz M, Brown $\mathrm{J}$ et al. Vulvar intraepithelial neoplasia (VIN2/3): comparing clinical outcomes and evaluating risk factors for recurrence. Gynecol Oncol 2012; 127:312-315.

3. Farr A, Wang H, Kasher MS, Roman A. Relative enhancer activity and transforming potential of authentic human papillomavirus type 6 genomes from benign and malignant lesions. J Gen Virol 1991;72:519-526.

4. Insinga RP, Liaw KL, Johnson LG, Madeleine MM. A systematic review of the prevalence and attribution of human papillomavirus types among cervical, vaginal, and vulvar precancers and cancers in the United States. Cancer Epidemiol Biomakers Prev 2008; 17:1611-1622.

5. Sutton BC, Allen RA, Moore WE, Dunn ST. Distribution of human papillomavirus genotypes in invasive squamous carcinoma of the vulva. Modern Pathology 2008;21:345-354.

6. De Vuyst H, Clifford GM, Nascimento MC, Madeleine MM, Franceschi S. Prevalence and type distribution of human papillomavirus in carcinoma and intraepithelial neoplasia of the vulva, vagina, and anus: a meta-analysis. Int J Cancer 2009;124:16261636. 
7. Tsimplaki E, Argyri E, Michala L, Kouvousi M, Apostolaki A, Magiakos G, et al. Human papillomavirus genotyping and E6/E7 mRNA expression in greek women with intraepithelial neoplasia and squamous cell carcinoma of the vagina and vulva. J Oncol 2012; 2012: 893275.

8. Lörincz AT, Quinn AP, Goldsborough MD, McAllister P, Temple GF. Human papillomavirus type 56: a new virus detected in cervical cancers. J Gen Virol 1989;70:3099-3104.
9. Dreyer L, Faurshou M, Mogensen M, Jacobsen S. High incidence of potentially virus-induced malignancies in systemic lupus erythematosus. Arthritis \& Reumatism 2011;63:3032-3037.

10. Yu SL, Chan PK, Wong CK, Szeto CC, Ho SC, So $\mathrm{K}$, et al. Antagonist-mediated down-regulation of toll-like receptors increases the prevalence of human papillomavirus infection in systemic lupus erythematosus. Arthritis Res Ther 2012;14:R80.

DOI: $10.5455 / 2320-1770 . i j r \operatorname{cog} 20130938$

Cite this article as: Seo S, Honda KI, Nakano Y, Umesaki N, Iwasa Y, Tanaka I, Iwai Y, Yasui T, Sumi T, Koyama M. Vulvar microinvasive squamous cell carcinoma arising in vulvar intraepithelial neoplasia 3 complicated by genital warts and systemic lupus erythematosus: a case report. Int J Reprod Contracept Obstet Gynecol 2013;2:437-40. 